\title{
APPROXIMATE CONTROLLABILITY RESULTS FOR IMPULSIVE LINEAR FUZZY STOCHASTIC DIFFERENTIAL EQUATIONS UNDER NONLOCAL CONDITIONS
}

\author{
S.Narayanamoorthy ${ }^{1}$ and S.Sowmiya ${ }^{2}$ \\ ${ }^{1}$ Department of Mathematics, \\ ${ }^{2}$ Department of Applied Mathematics \\ Bharathiar University, Coimbatore-641 046, INDIA.
}

\begin{abstract}
In this paper, the approximate controllability of impulsive linear fuzzy stochastic differential equations with nonlocal conditions in Banach space is studied. By using the Banach fixed point theorems, stochastic analysis, fuzzy process and fuzzy solution, some sufficient conditions are given for the approximate controllability of the system.
\end{abstract}

\section{KEY WORDS}

Approximate controllability, impulsive linear fuzzy stochastic differential equations, fuzzy solution, fixed point theorems, nonlocal conditions.

2010 MSC: $03 E 72,39 A 50,93 B 05,49 N 25,47 H 10$

\section{INTRODUCTION}

Differential equations have been used in modeling the dynamics of changing processes. A great dense of the modeling, development has been accompanied by a rich theory of differential equations. Freshly, stochastic differential equations (SDEs) occur in the mathematical modeling of various fields in physics and engineering science. Among them, several properties of SDEs such as existence, controllability, and stability are studied for the linear and nonlinear equations. But in many situations, it is useful to investigate the linear stochastic differential equations directly discussed by Daniel [2]. The properties and applications are presented in [5].

Fuzzy set theory introduced by Zadeh [19] is a generalization of abstract set theory. Semwal et. al [14] studied the less computationally intensive fuzzy logic based controller for humanoid push recovery. The theory of Impulsive differential equations have been developed in modeling impulsive problems in physics, population dynamics, ecology, biological systems, robotics, optimal control and so forth. In $[6,7,9]$ these types of impulsive effects and differential systems were studied. The nonlocal condition, which is a generalization of the classical initial condition, was motivated by physical problems. Among others, we refer to the papers in $[1,4,13]$. In general, fixed point theorems are very useful classes of result that give us the conditions under which for some function $\mathrm{f}$. The Sadovskii fixed point theorem and the theory of strongly continuous cosine families of operators are used to study the sufficient conditions for the controllability of the system considered and that are discussed in $[3,14,17]$. 
The concept of the controllability has played a central role during the history of modern control theory. The approximate controllable systems are more common and very often approximate controllability is completely sufficient in applications. Therefore, it is important and necessary to study the weaker concept of controllability, namely approximate controllability for linear and nonlinear differential systems. In $[10,11]$ these types of controllability properties and conditions were discussed.

In the literature there are only some papers that deal with the approximate controllability of fuzzy differential and linear fuzzy differential systems, likewise approximate controllability of second order stochastic systems as discussed in [17]. Subalakshmi and Balachandran [16] studied the nonlinear stochastic impulsive integrodifferential system's approximate controllability. Mahmudov and Zorlu [8] discussed the concept of the approximate controllability of semilinear neural systems in Hilbert space. Narayanamoorthy and Sowmiya [12] presented the concept of the approximate controllability result for nonlinear impulsive neural fuzzy stochastic differential equation with nonlocal conditions. It should be mentioned that there is no work done on the approximate controllability of linear fuzzy stochastic differential equations. Motivated by the above consideration in this paper, we examine the approximate controllability for impulsive linear fuzzy stochastic differential equation with nonlocal conditions.

The rest of the paper is organized as follows. In the following section, we give the necessary preliminaries, definitions, lemmas and theorems. In section 3, we deduce the main result on the approximate controllability result for linear fuzzy stochastic differential systems. Finally, in Section 4 includes the conclusion.

\section{PROBLEM FORMULATION AND PRELIMINARIES}

Here, first we define some properties, theorems and lemma's and also recalls some basic definitions which are all used in this paper. A fuzzy set of $\mathbf{R}^{\mathbf{n}}$ is a function $\mathbf{u} \mathbf{R}^{\mathbf{n}} \rightarrow[0,1]$. For each fuzzy set $\mathrm{u}$, we denote by $[\mathrm{u}]^{\alpha}=\mathrm{x} \in \mathrm{R}^{\mathrm{n}}$ : $\mathrm{u}(\mathrm{x}) \geq \alpha$ for any $\alpha \in[0,1]$, its $\alpha$-level set.

Let $\mathrm{u}, \mathrm{v}$ be fuzzy sets of $\mathrm{R}^{\mathbf{n}}$. It is well known that $\left.[\mathrm{u}]^{\boldsymbol{\alpha}}={ }_{\mathrm{v}}\right]^{\boldsymbol{\alpha}}$ for each $\alpha \in[0,1]$ implies $\mathrm{u}$ $=\mathrm{v}$. Let $\mathrm{E}^{\mathrm{n}}$ denote the collection of all fuzzy sets of $\mathrm{R}^{\mathrm{n}}$ that satisfies the following conditions:

- $\mathrm{u}$ is normal

- fuzzy convex

- upper semicontinuous and

- $[\mathrm{u}]^{\mathrm{O}}$ is compact.

We call $\mathrm{u} \in \mathbf{E}^{\mathbf{n}}$ is an n-dimensional fuzzy number.

Definition 2.1. If $\mathrm{u} \in \mathrm{E}^{\mathbf{n}}$, and $[\mathrm{u}]^{\boldsymbol{\alpha}}$ is a hyperrectangle, that is, $[\mathrm{u}]^{\boldsymbol{\alpha}}$ can be represented by $\prod_{i=1}^{n}\left[u_{i l}^{\alpha}, u_{i r}^{\alpha}\right]$, that is $\left[u_{1 l^{\alpha}}^{\alpha}, u_{1 r}^{\alpha}\right] \times\left[u_{2 l}^{\alpha}, u_{2 r}^{\alpha}\right] \times \ldots \times\left[u_{n l^{\alpha}}^{\alpha}, u_{n r}^{\sigma}\right]$ for every $\alpha \in[0,1]$, where $u_{i l}^{\alpha} u_{i r}^{\alpha} \in \mathrm{R}$ with $u_{i l}^{\alpha} \leq u_{i r}^{\alpha}$ when $\alpha \in(0,1], \mathrm{i}=1,2, \ldots, \mathrm{n}$, then we call $\mathrm{u}$ is a fuzzy $\mathrm{n}$-cell number. We denote the collection of all fuzzy $\mathrm{n}$-cell numbers by $\mathrm{L}\left(\mathrm{E}^{\mathrm{n}}\right)$. 


\section{Theorem 2.2.}

For any $\mathrm{u} \in \mathrm{L}\left(\mathrm{E}^{\mathrm{n}}\right)$ with $[\mathrm{u}]^{\alpha}=\prod_{i=1}^{n}\left[u_{i L}^{\alpha}, u_{i r}^{\alpha}\right](\alpha \in[0,1])$, there exist a unique $\left(\mathrm{u}_{1}, \mathrm{u}_{2}, \ldots, \mathrm{u}_{\mathrm{n}}\right) \in\left(\mathrm{E}^{\mathrm{n}}\right)$ such that $[\mathrm{u}]^{\alpha}=\left[u_{i L}^{\alpha}, u_{i r}^{\alpha}\right](\mathrm{i}=1,2, \ldots, \mathrm{n}$ and $\alpha \in[0,1])$.

Conversely, for any $\left(\mathrm{u}_{1}, \mathrm{u}_{2}, \ldots, \mathrm{u}_{\mathrm{n}}\right) \in\left(\mathrm{E}^{\mathrm{n}}\right)$ with $[\mathrm{u}]^{\alpha}=\left[u_{i L}^{\sigma}, u_{i r}^{\alpha}\right](\mathrm{i}=1,2, \ldots, \mathrm{n}$ and $\alpha \in[0,1])$, there exit a unique $\mathrm{u} \in \mathrm{L}\left(\mathrm{E}^{\mathrm{n}}\right)$ such that $[\mathrm{u}]^{\alpha}=\prod_{i=1}^{n}\left[u_{i l}^{\alpha}, u_{i r}^{\alpha}\right](\alpha \in[0,1])$.

Definition 2.3. The complete metric $\mathrm{D}_{\mathrm{L}}$ on $\left(E_{N}^{i}\right)^{\mathrm{n}}$ is defined by

$$
D_{L}(u, v)=\sup _{0<\alpha \leq 1} d_{L}\left([u]^{\alpha},[v]^{\alpha}\right)=\sup _{0<\alpha \leq 1} \max _{1 \leq i \leq n}\left\{\left|u_{i l}^{\alpha}-v_{i l}^{\alpha}\right|,\left|u_{i r}^{\alpha}-v_{i r}^{\alpha}\right|\right\}
$$

for any u, ve $\mathrm{L}\left(E_{N}^{i}\right)^{\mathrm{n}}$, which satisfied $d_{L}(u+w, v+w)=d_{L}(u+v)$.

Definition 2.4. Let $\left.u, v \in C([0, T]):\left(E_{N}^{i}\right)^{n}\right)$, then

$$
H_{1}(u, v)=\sup _{0 \leq t \leq T} D_{L}(u(t), v(t))
$$

Definition: 2.5 [17]. A stochastic process $x$ is said to be a mild solution of (3.1) if the following conditions are satisfied:

a. $\quad \mathrm{x}(\mathrm{t}, \omega)$ is a measurable function from $J \times \Omega$ to $\mathrm{H}$ and $x(t)$ is $F t$-adapted,

b. $\mathrm{E}\|\mathrm{x}(\mathrm{t})\|^{2}<\infty$ for each $\mathrm{t} \in \mathrm{J}$,

c. $\Delta x\left(\tau_{i}\right)=x\left(\tau_{i}^{+}\right)-x\left(\tau_{i}^{-}\right)=I_{i}\left(x\left(\tau_{i}\right)\right) . \quad x \in X, 1 \leq i \leq m$.

d. For each $\mathrm{u} \in \mathrm{L}_{2}^{F}(\mathrm{~J}, \mathrm{U})$, the process $x$ satisfies the following integral equation

$$
\begin{aligned}
x(t)=U( & t, 0)\left[x^{1}-\mu(x)\right]+\int_{0}^{t} U(t, s) B u(s) d s \\
& +\int_{0}^{t} U(t, s) f(s, x(s)) d s+\int_{0}^{t} U(t, s) g(s, x(s)) d W(s) \\
& +\sum_{0 \leq t_{i} \leq t} U\left(t, t_{i}\right) I_{i}\left(x\left(t_{i}^{-}\right)\right), t \in J .
\end{aligned}
$$

Definition: 2.6. Let $x, y \in C\left(I: E^{n}\right)$, here $I$ be a real interval. A mapping $x: I \rightarrow E_{N}$ is called a fuzzy process. We denote,

$$
[x(t)]^{\alpha}=\left[x_{l}^{\alpha}(t), x_{r}^{\alpha}(t)\right], t \in I, 0<\alpha \leq 1 .
$$

The derivative $x^{\prime}(t)$ of a fuzzy process $x$ is defined by

$$
\left[x^{\prime}(t)\right]^{\alpha}=\left[\left(x_{l}^{\alpha}\right)^{\prime}(t),\left(x_{r}^{\alpha}\right)^{\prime}(t)\right], t \in I, 0<\alpha \leq 1
$$

provided that is equation defines a fuzzy $x^{\prime}(t) \in E_{N}$. 
Definition: 2.7. The fuzzy process $x:[0, T] \rightarrow\left(E_{N}^{i}\right)^{n}$ with $\alpha$-level set $[x(t)]^{\alpha}=\prod_{i=1}^{n}\left[x_{i l}^{\alpha}, x_{i r}^{\alpha}\right]$ is a fuzzy solution of (3.1) with homogenous term iff

$$
\begin{aligned}
& \left(x_{i l}^{a}\right)^{\prime}(t)=\min \left\{A_{i l}^{a}(t) x_{i k}^{a}: j, k=l, r\right\} \\
& \left(x_{i r}^{a}\right)^{\prime}(t)=\min \left\{A_{i r}^{a}(t) x_{i k}^{a}: j, k=l, r\right\}
\end{aligned}
$$

and

$$
x_{i l}^{a}(0)+g_{i l}^{a}\left(x_{i l}^{a}\right)=x_{0_{i l}}^{a}, x_{i r}^{a}(0)+g_{i r}^{a}\left(x_{i r}^{a}\right)=x_{0_{i r}}^{a}, i=1,2, \ldots, n \text {. }
$$

Lemma 2.8. Assume that $x \in B_{h}^{\prime}$, then for $\mathbf{t} \in \mathbf{I}, \mathbf{x} \in \mathrm{B}_{\mathrm{h}}$. Moreover,

$$
l|x(t)| \leq\left\|x_{t}\right\|_{B h} \leq\left\|x_{0}\right\|_{B h}+l \sup _{s \in[0, t]}\|x(s)\|
$$

where $l=\int_{-\infty}^{0} h(t) d t<\infty$

Note 2.9. Define $B_{h}^{\prime \prime}=z \in B_{h}^{\prime}: z_{0}=0 \in B_{h}$. For any $z \in B_{h}^{\prime \prime}$,

$$
\begin{aligned}
\|z\|_{B h}^{\prime \prime} & =\left\|z_{0}\right\|_{B h}+\sup |z(s)|: s \in[0, b] \\
& =\sup |z(s)|: s \in[0, b],
\end{aligned}
$$

and thus $\left(B_{h}^{\prime \prime},\|\cdot\|\right)$ is a Banach space. Set

$$
B_{r}=z \in P C((-\infty, b], X):\|z(t)\|_{B h}^{\prime} \leq r, \quad 0 \leq t \leq b .
$$

Clearly $\mathrm{B}_{\mathbf{r}}$ is a nonempty, bounded, convex and closed set in $B_{h}^{\prime}$. Then for any $\mathrm{z} \in \mathrm{B}_{\mathbf{r}}$, from Lemma [2.4], we have

$$
\begin{gathered}
\left\|z_{t}+y_{t}\right\|_{B h} \leq\left\|z_{t}\right\|_{B h}+\left\|y_{t}\right\|_{B h} \\
\leq\left\|z_{0}\right\|_{B h}+l \sup _{s \in[0, b]}|z(s)|+\left\|y_{0}\right\|_{B h}+l \sup _{s \in[0, b]}|y(s)| \\
\leq l\left(r+M_{1}|\phi(0)|\right)+\|\phi\|_{B h}=r^{\prime}
\end{gathered}
$$

for each $\mathbf{t} \in \mathbf{J}, \mathrm{z} \in \mathrm{B}_{\mathbf{r}}$, we have by above equation and (A2)

$$
\begin{aligned}
\sup _{t \in J}|z(t)+y(t)| & \leq l^{-1}\left\|z_{t}+y_{t}\right\|_{B h} \leq l^{-1} r^{\prime}, \\
\mid I_{k}\left(z\left(t_{k}^{+}\right)+z\left(t_{k}^{-}\right) \mid\right. & \leq d_{k}\left(\sup _{t \in J}|z(t)+y(t)|\right) \\
& \leq d_{k}\left(l^{-1} r^{\prime}\right), k=1,2, \ldots, m .
\end{aligned}
$$


Let $\mathrm{x}_{\mathrm{b}}\left(\mathrm{x}_{0} ; \mathrm{u}\right)$ be the state value of (3.1) at terminal time $\mathrm{b}$ corresponding to the control $\mathrm{u}$ and initial value $\mathrm{x}_{0}=\varphi \in \mathrm{B}_{\mathrm{h}}$. Introduce the set $\mathrm{R}\left(\mathrm{b}, \mathrm{x}_{0}\right)=\mathrm{x}_{\mathrm{b}}\left(\mathrm{x}_{0} ; \mathrm{u}\right)(0)$ : $\mathrm{u}(\cdot) \in \mathrm{L}^{2}(\mathrm{~J}, \mathrm{U})$ which is called the reachable set at terminal time $\mathrm{b}$, its closure in $\mathrm{X}$ is denoted by $\overline{R\left(b, x_{0}\right)}$.

Definition 2.10. If $\overline{R\left(b, x_{0}\right)}=X$ then the System (3.1) is approximately controllable on the interval J. It is convenient at this point to define operators

$\Gamma_{0}^{\alpha}=\int_{0}^{\alpha} U(a, s) B B^{*} U^{*}(a, s) d s$

$R\left(\alpha, \Gamma_{0}^{\alpha}\right)=\left(\alpha I, \Gamma_{0}^{\alpha}\right)^{-1}$

(D): $\alpha R\left(\alpha, \Gamma_{00}^{\alpha}\right) \rightarrow 0$ as $\alpha \rightarrow 0^{+}$is strong operator topology. It is known that assumption (D) holds iff the linear system

$\dot{x}(t)=A x(t)+B u(t), x(0)=x_{0}$

is approximately controllable on $\mathbf{J}$.

The following theorem gives a formula for a control transferring the initial state $\mathrm{x}_{0}$ to some neighborhood of $\mathrm{x}_{\mathrm{a}}$ at time $\mathrm{a}$.

\section{Theorem 2.11.}

For arbitrary $\mathrm{x}_{\mathrm{a}} \in \mathrm{X}$, the control

$$
u(t)=B^{*} S^{*}(a-t) R\left(\alpha, \Gamma_{0}^{\alpha}\right) p(x(\cdot))
$$

Where

$$
\begin{aligned}
& p(x(\cdot))=x_{a}-S(a)\left[x_{0}-g(x)\right]-\int_{0}^{a} S(a-s) F(s) x(s) d s \\
& \quad-\sum_{k=1}^{n} S(a-s) \int_{0}^{a} S(a-s) G_{k}(s) x(s) d W_{k}(s)-\sum_{j=1}^{m} S\left(a-t_{j}\right)\left(I_{j}\right)\left(x\left(t_{j}^{-}\right)\right) \\
& x(a)=x_{a}-\alpha\left(\alpha I+\Gamma_{0}^{a}\right)^{-1}\left[S(a)\left[x_{0}-g(x)\right]-\int_{0}^{a} S(a-s) F(s) x(s) d s\right. \\
& \left.\quad-\sum_{k=1}^{n} S(a-s) \int_{0}^{a} S(a-s) G_{k}(s) x(s) d W_{k}(s)-\sum_{j=1}^{m} S\left(a-t_{j}\right)\left(I_{j}\right)\left(x\left(t_{j}^{-}\right)\right)\right] .
\end{aligned}
$$




\section{MAIN RESULT}

In this section, we verify that the approximate controllability result for impulsive linear fuzzy stochastic differential equation condition by using Ban ach fixed point theorem. Let us consider the impulsive linear fuzzy stochastic differential equation in $\left(E_{N}^{i}\right)^{n}$.

$$
\begin{aligned}
& d x(t)=A x(t)+F(t) x(t) d t+B u(t) d t+\sum_{k=1}^{n} G_{k}(t) x(t) d W_{k}(t) \\
& \Delta x\left(t_{j}\right)=x\left(t_{j}^{+}\right)-x\left(t_{j}^{-}\right)=I_{j}\left(x\left(t_{j}\right)\right), x \in X \\
& x(0)+g(x)=x_{0} \in\left(E_{N}^{i}\right)^{n}
\end{aligned}
$$

With fuzzy coefficient $A:[0, T] \rightarrow\left(E_{N}^{i}\right)^{n}, x_{0} \in\left(E_{N}^{i}\right)^{n}$ is a initial value, and $u:[0, T] \rightarrow\left(E_{N}^{i}\right)^{n}$ is a control function.

To establish the result, we introduce the following assumptions on systems (3.1).

$\left(\mathrm{A}_{1}\right) . \mathrm{S}(\mathrm{t})$ is a fuzzy number satisfying for $y \in\left(E_{N}^{i}\right)^{n}(d / d t) S(t) y \in C^{\prime}\left(I:\left(E_{N}^{i}\right)^{n}\right.$, $\cap C^{\prime}\left(I:\left(E_{N}^{i}\right)^{n}\right)$ then,

where,

$$
(d / d t) S(t) y=A[S(t) y]=S(t) A y, t \in I
$$

$$
[S(t)]^{\alpha}=\prod_{i=1}^{n}\left[S_{i}(t)\right]^{\alpha}=\prod_{i=1}^{n}\left[S_{i l}{ }^{\alpha}(t), S_{i r}{ }^{\alpha}(t)\right]
$$

and $S_{i j}^{\alpha}(t)(j=l, r)$ is continuous with $\left|S_{i j}{ }^{\alpha}(t)\right| \leq C, C>0$, for all $t \in I=[0, T]$

(A2 ). The nonlinear fuzzy function $F:[0, T] \times\left(E_{N}^{i}\right)^{n} \rightarrow\left(E_{N}^{i}\right)^{n}$ and $g:\left(E_{N}^{i}\right)^{n} \rightarrow\left(E_{N}^{i}\right)^{n}$ is continuous function and both are satisfied the global Lipschitz condtions, such that,

$d_{L}\left([F x(s)]^{\alpha},[F y(s)]^{\alpha}\right) \leq f d_{L}\left([x(s)]^{\alpha},[y(s)]^{\alpha}\right)$

and

$$
d_{L}\left([g x(s)]^{\alpha},[g y(s)]^{\alpha}\right) \leq b d_{L}\left([x(.)]^{\alpha},[y(.)]^{\alpha}\right)
$$

for all $x(s), y(s), x(),. y(.) \in\left(E_{N}^{i}\right)^{n}, \mathrm{f}$ and $\mathrm{b}$ are a finite positive constants.

(A3 ) The fuzzy continuous function $G_{k}:[0, T] \times\left(E_{N}^{i}\right)^{n} \rightarrow\left(E_{N}^{i}\right)^{n}$ is a strongly measurable and satisfied the lipschitz condition, such that

$$
d_{L}\left(\left[G_{k} x(s)\right]^{\alpha},\left[G_{k} y(s)\right]^{\alpha}\right) \leq g d_{L}\left([x(s)]^{\alpha},[y(s)]^{\alpha}\right)
$$

for all $x(s), y(s) \in\left(E_{N}^{i}\right)^{n}$ and $\mathrm{k}=1,2, \ldots, \mathrm{n}$ : $\mathrm{g}$ is positive constant. 
(A4) The function $I_{i}:\left(E_{N}^{i}\right)^{n} \rightarrow\left(E_{N}^{i}\right)^{n}$ is a compact and there exist a positive constant $\mathrm{d}$ such that

$$
d_{L}\left(\left[I_{i} x(s)\right]^{\alpha},\left[I_{i} y(s)\right]^{\alpha}\right) \leq d d_{L}\left([x(.)]^{\alpha},[y(.)]^{\alpha}\right)
$$

for all $x(),. y(.) \in\left(E_{N}^{i}\right)^{n}$

$\left(\mathrm{A}_{5}\right) . c b(1+T+c T)+f T(1+c T)+g T(1+c T)+d(1+c T)<1$.

From Note 2.6 and hypotheses (A1), equation (3.1) can be expressed as

$$
\begin{aligned}
& x(t)=S(t)\left[x_{0}-g(x)\right]+\int_{0}^{t} S(t-s) B u(s) d s+\int_{0}^{t} S(t-s) F x(s) d s+\sum_{k=1}^{n} \int_{0}^{t} S(t-s) G_{k} x(s) d W_{k}(s) \\
& \Delta x\left(t_{j}\right)=x\left(t_{j}^{+}\right)-x\left(t_{j}^{-}\right)=I_{j}\left(x\left(t_{j}\right)\right) \\
& x(0)+g(x)=x_{0}
\end{aligned}
$$

\section{Theorem 3.1}

Let $\mathrm{T}>0$, if hypotheses (A1 ) $-\left(\mathrm{A}_{2}\right)$ are hold, then for every $x_{0} \in\left(E_{N}^{i}\right)^{n}$, equation (3.1)(u $\equiv 0)$ have a unique fuzzy $x \in C\left([0, T]:\left(E_{N}^{i}\right)^{n}\right)$.

\section{Theorem 3.2}

Condition (A1 ) - (A4) and (D) are satisfied. Then the system (3.1) is approximate controllability on $\mathbf{J}$.

\section{Proof:}

Let $\widetilde{\mathrm{x}}^{\beta}(\cdot)$ be a fixed point of $\Phi$ and any fixed point of $\Phi$ is a mild solution of (3.1) on [0, b]. By theorem 2.7, the control

$$
\tilde{u}^{\beta}(t)=B^{*} S^{*}(a-t) \tilde{R}\left(\beta, \Gamma_{o}^{a}\right)\left[P\left(\tilde{x}^{\beta}\right)\right]^{\alpha}
$$

which satisfies

$$
\begin{gathered}
{\left[\tilde{x}^{\beta}(a)\right]^{\alpha}=\left[x_{\alpha}-\beta \tilde{R}\left(\beta, \Gamma_{o}^{a}\right)\left[x_{\alpha}-S_{i l}(a)\right]\left[x_{0}-g_{i l}(x)\right]-\int_{0}^{a} S_{i l}^{\alpha}(a-s) F_{i l}^{\alpha}\left(\tilde{x}^{\beta}\right) d s\right.} \\
-\sum_{k=1}^{n} \int_{0}^{a} S_{i l}^{\alpha}(a-s)\left(G_{k}\right)_{i l}^{\alpha}\left(\tilde{x}_{s}^{\beta}\right) d W_{k}(S)-\sum_{j=1}^{m} S_{i l}^{\alpha}\left(a-t_{j}\right)\left(I_{j}\right)\left(x\left(t_{j}^{-}\right)\right), \\
x_{\alpha}-S_{i l}^{\alpha}(a)\left[x_{0}-g_{i r}(x)\right]-\int_{0}^{a} S_{i r}^{\alpha}(a-s) F_{i r}^{\alpha}\left(\tilde{x}^{\beta}\right) d s \\
\left.-\sum_{k=1}^{n} \int_{0}^{a} S_{i r}^{\alpha}(a-s)\left(G_{k}\right)_{i r}^{\alpha}\left(\tilde{x}_{s}^{\beta}\right) d W_{k}(S)-\sum_{j=1}^{m} S_{i r}^{\alpha}\left(a-t_{j}\right)\left(I_{j}\right)\left(x\left(t_{j}^{-}\right)\right)\right]
\end{gathered}
$$

where, 
International Journal of Fuzzy Logic Systems (IJFLS) Vol.5, No.4, October 2015

$$
\begin{aligned}
{\left[P\left(\tilde{x}^{\beta}\right)\right]^{\alpha}=} & {\left[x_{\alpha}-S_{i l}(a)\right]\left[x_{0}-g_{i l}(x)\right]-\int_{0}^{a} S_{i l}^{\alpha}(a-s) F_{i l}^{\alpha}\left(\tilde{x}_{s}^{\beta}\right) d s } \\
& -\sum_{k=1}^{n} \int_{0}^{a} S_{i l}^{\alpha}(a-s)\left(G_{k}\right)_{i l}^{\alpha}\left(\tilde{x}_{s}^{\beta}\right) d W_{k}(S)-\sum_{j=1}^{m} S_{i l}^{\alpha}\left(a-t_{j}\right)\left(I_{j}\right)\left(x\left(t_{j}^{-}\right)\right), \\
& x_{\alpha}-S_{i r}^{\alpha}(a)\left[x_{0}-g_{i r}(x)\right]-\int_{0}^{a} S_{i r}^{\alpha}(a-s) F_{i r}^{\alpha}\left(\tilde{x}^{\beta}\right) d s \\
& \left.-\sum_{k=1}^{n} \int_{0}^{a} S_{i r}^{\alpha}(a-s)\left(G_{k}\right)_{i r}^{\alpha}\left(\tilde{x}_{s}^{\beta}\right) d W_{k}(S)-\sum_{j=1}^{m} S_{i r}^{\alpha}\left(a-t_{j}\right)\left(I_{j}\right)\left(x\left(t_{j}^{-}\right)\right)\right]
\end{aligned}
$$

By $(\mathrm{A} 2)-(\mathrm{A} 4)$

$$
\begin{aligned}
& d_{L}\left(\left[F \tilde{x}_{s}^{\beta}\right]^{\alpha},[F(s)]^{\alpha}\right) \leq f d_{L}, d_{L}\left(\left[\left(G_{k}\right) \tilde{x}_{s}^{\beta}\right]^{\alpha},\left[\left(G_{k}\right)(s)\right]^{\alpha}\right) \leq g d_{L} \\
& d_{L}\left(\left[g \tilde{x}_{s}^{\beta}\right]^{\alpha},[g(s)]^{\alpha}\right) \leq b d_{L}
\end{aligned}
$$

Here, the sequence $F_{i l}^{\alpha}\left(\tilde{x}_{s}^{\beta}\right),\left(G_{k}\right)_{i l}^{\alpha}\left(\tilde{x}_{s}^{\beta}\right)$ and $g_{i l}^{\alpha}\left(\tilde{x}_{s}^{\beta}\right)$ are bounded in $\left(E_{N}^{i}\right)^{n}$. And $F_{i l}^{\alpha}(s),\left(G_{k}\right)_{i l}^{\alpha}(s)$ and $g_{i l}^{\alpha}(s)$ are all weakly convergent subsequence that are bounded in $\left(E_{N}^{i}\right)^{n}$, respectively. Take

$$
\begin{aligned}
\tilde{\omega} & =\left[x_{\alpha}-S_{i l}(a)\right]\left[x_{0}-g_{i l}^{\alpha}(x)\right]-\int_{0}^{a} S_{i l}^{\alpha}(a-s) F_{i l}^{\alpha}(s) d s \\
& -\sum_{k=1}^{n} \int_{0}^{a} S_{i l}^{\alpha}(a-s)\left(G_{k}\right)_{i l}^{\alpha}(s) d W_{k}(S)-\sum_{j=1}^{m} S_{i l}^{\alpha}\left(a-t_{j}\right)\left(I_{j}\right)\left(x\left(t_{j}^{-}\right)\right), \\
& x_{\alpha}-S_{i r}^{\alpha}(a)\left[x_{0}-g_{i r}(x)\right]-\int_{0}^{a} S_{i r}^{\alpha}(a-s) F_{i r}^{\alpha}(s) d s \\
& \left.-\sum_{k=1}^{n} \int_{0}^{a} S_{i r}^{\alpha}(a-s)\left(G_{k}\right)_{i r}^{\alpha}(s) d W_{k}(S)-\sum_{j=1}^{m} S_{i r}^{\alpha}\left(a-t_{j}\right)\left(I_{j}\right)\left(x\left(t_{j}^{-}\right)\right)\right] .
\end{aligned}
$$

It follows by the compactness of the operators

$$
\begin{aligned}
q(.) \rightarrow \int_{0}^{a} U(., s) q(s) d s: & L^{2}(J, X) \rightarrow C(J, X), \text { we obtain that } \\
\qquad\left[P\left(\tilde{x}^{\gamma}\right)\right]^{\alpha}-\tilde{\omega} \| & \leq\left\|\int_{0}^{a} S_{i j}^{\alpha}(a-s)\left[F_{i j}^{\alpha}\left(\tilde{x}_{s}^{\beta}\right), F_{i j}^{\alpha}(s)\right] d s\right\| \\
& +\left\|\sum_{k=1}^{n} \int_{0}^{a} S_{i l}^{\alpha}(a-s)\left[\left(G_{k}\right)_{i l}^{\alpha}\left(\tilde{x}_{s}^{\beta}\right),\left(G_{k}\right)_{i l}^{\alpha}(s)\right] d W_{k}(S)\right\| \rightarrow 0 \text { as } \beta \rightarrow 0^{+}
\end{aligned}
$$

where, $\mathbf{j}=1$, r. By Definition 


$$
\begin{aligned}
\left\|\left[\tilde{x}^{\gamma}(a)\right]^{\alpha}-x_{\alpha}\right\| & =\left\|\beta \tilde{R}\left(\beta, \Gamma_{o}^{a}\right)\left[P\left(\tilde{x}^{\beta}\right)\right]^{\alpha}\right\| \\
& =\left\|\beta \tilde{R}\left(\beta, \Gamma_{o}^{a}\right)\left(\left[P\left(\tilde{x}^{\beta}\right)\right]^{\alpha}-\tilde{\omega}+\tilde{\omega}\right)\right\| \\
& \leq\left\|\beta \tilde{R}\left(\beta, \Gamma_{o}^{a}\right) \tilde{\omega}\right\|+\left\|\beta \tilde{R}\left(\beta, \Gamma_{o}^{a}\right)\left[P\left(\tilde{x}^{\beta}\right)\right]^{\alpha}-\tilde{\omega}\right\| \\
& \leq\left\|\beta \tilde{R}\left(\beta, \Gamma_{o}^{a}\right) \tilde{\omega}\right\|+\left\|\left[P\left(\tilde{x}^{\beta}\right)\right]^{\alpha}-\tilde{\omega}\right\| \rightarrow 0 \text { as } \beta \rightarrow 0^{+}
\end{aligned}
$$

ie., $\left\|\left[\tilde{x}^{\gamma}(a)\right]^{\alpha}-x_{\alpha}\right\| \rightarrow 0$. ie., this proves the approximate controllability of (3.1).

\section{CONCLUSION}

The article addresses the approximate controllability results for impulsive linear fuzzy stochastic differential equations with nonlocal conditions in Banach space. By using stochastic analysis, fuzzy process, fuzzy solution, Banach fixed point theorems and some sufficient conditions for the approximate controllability of the linear fuzzy stochastic control system are formulated and proved under the assumptions that are related linear system is approximate controllable.

\section{ACKNOWLEDGMENTS}

The authors would like to thank Dr.K.Balachandran, Professor and Head, Department of Mathematics Bharathiar University for lending his support to complete this article.

\section{REFERENCES}

[1] K. Balachandran and S. Ilamaran, Existence and Uniqueness of Mild and Strong Solutions of a Semilinear Evolution Equation with Nonlocal Condition, Indian Journal of Pure and Applied Mathematics, (1994) Vol. 25, 411-418.

[2] Daniel Ocone and Etienne Pardoux, Linear Stochastic Differential Equations with boundary Conditions, Probability Theory and Related Fields, (1989) 82, 489-526.

[3] Dimplekumar N. Chalishajar1, Falguni S. Acharya, Controllability of Neutral Impulsive Differential Inclusions with Non-Local Conditions, Applied Mathematics, (2011) 2, 1486-1496.

[4] M. Guo, X. Xue, R. Li, Controllability of Impulsive Evolution Inclusions with Nonlocal Conditions, Journal of Optimization Theory and Applications, (2004) Vol. 120, No. 2, 355-374.

[5] Jai Heui Kim, On Fuzzy Stochastic Differential Equations, J. Korean Math. Soc. (2005), 42 No. 1, 153-169.

[6] V. Lakshmikantham, D.D. Bainov and P.S. Simeonov, Theory of impulsive differential equations, World Scienti_c, Singapore, (1989).

[7] Lei Zhang, Yongsheng Ding, Tong Wang, Liangjian Hu and Kuangrong Hao, Controllability of Second-Order Semilinear Impulsive Stochastic Neutral Functional Evolution Equations, Mathematical Problems in Engineering, Volume 2012, Article ID 748091, 1-13.

[8] N. I. Mahmudov and S. Zorlu, Approximate controllability of Semilinear neutral systems in hilbert spaces, Journal of Applied Mathematics and Stochastic Analysis, (2003), 16:3 233-242.

[9] M. Mallika Arjunana, V. Kavithab, S. Selvi, Existence results for impulsive differential equations with nonlocal conditions via measures of noncompactness, J. Nonlinear Sci. Appl. (2012) 5, 195-205.

[10] S. Narayanamoorthy, M. Nagarajan, Controllability for the Nonlinear Integrodifferential Equation with Nonlocal Condition, Research journal of science and tech, 5 (2012), 25-31.

[11] S. Narayanamoorthy, S. Sowmiya, Controllability Result for Nonlinear Impulsive Neutral Fuzzy Stochastic Differential Equations in Banach Space, Dynamics of Continuous, Discrete and Impulsive Systems (to appear).

[12] S. Narayanamoorthy, S. Sowmiya, Approximate Controllability result for nonlinear Impulsive Neutral fuzzy stochastic differential equation with nonlocal conditions Advances in Difference Equations, doi:10.1186/s13662-015-0454-2. 
[13] S.K. Ntouyas and P.C. Tsamatos, Global Existence for Semilinear Evolution Equations with Nonlocal Conditions, Journal of Mathematical Analysis and Applications, (1997) Vol. 210, 679- 687.

[14] B. Sadaovskii, On a fixed point principle, Functional Analysis and Applications, (1967) 2, 151-153.

[15] Semwal, Vijay Bhaskar, Praven Chakraborty and G.C. Nandi, Less Computationally intensive fuzzy logic (type-1)-based controller for humanoid push recovery, Robotics and Autonomous Systems,(2015) 63, 122-135.

[16]R. Subalakshmi and K. Balachandran, Approximate controllability of nonlinear stochastic impulsive integrodifferential systems in hilbert spaces, Chaos, Solitons and Fractals, (2009) 42, 2035-2046.

[17] Urvashi Arora and N. Sukavanam, Approximate controllability of second order semilinear stochastic system with nonlocal conditions, Applied Mathematics and Computation, (2015) 258, 111-119.

[18] Young Chel Kwun, Jeong Soon Kim, Min Ji Park, and Jin Han Park, Nonlocal Controllability for the Semilinear Fuzzy Integrodifferential Equations in n-Dimensional Fuzzy Vector Space, Advances in Difference Equations, Volume 2009, Article ID 734090, 16 pages.

[19] L.A. Zadeh, Fuzzy sets, Inf. Control, (1965) 8, 338353. 10. 